\title{
Extracting and Combining Multimodal Directional Iris Features
}

\author{
Chul-Hyun Park ${ }^{1}$ and Joon-Jae Lee ${ }^{2}$ \\ ${ }^{1}$ School of Electrical and Computer Engineering, Purdue University, \\ West Lafayette, Indiana 47907-2035, USA \\ park95@purdue.edu \\ ${ }^{2}$ Dept. of Computer and Information Engineering, \\ Dongseo University, Busan, Korea \\ jjlee@dongseo.ac.kr
}

\begin{abstract}
In this paper, we deal with extracting and combining multimodal iris features for person verification. In multibiometric approaches, finding reasonably disjoint features and effective combining methods are crucial. The proposed method considers the directional characteristics of iris patterns as critical features, and first decomposes an iris image into several directional subbands using a directional filter bank (DFB), then generates two kinds of feature vectors from the directional subbands. One is the binarized output features of the directional subbands on multiple scales and the other is the blockwise directional energy features. The former is relatively robust to changes in illumination or image contrast because it uses the directional zero crossing information of the directional subbands, whereas the latter provides another form of rich directional information though it is a bit sensitive to contrast change. Matching is performed separately between the same kind of feature vectors and the final decision is made by combining the matching scores based on the accuracy of each method. Experimental results show that the two kinds of feature vectors used in this paper are reasonably complementary and the combining method is effective.
\end{abstract}

\section{Introduction}

Though human irises have been successfully used in some applications as a means for human identification [1], finding a method robust to various environmental situations such as changes in illumination or image contrast is still a challenging issue. Actually, the local and global brightness values of an iris image change according to the positions of various light sources and the image contrast also varies due to different focusing of the camera. To accomplish robustness to such changes, most conventional approaches use the quantized values of the transformed data or multi-resolution features [2-4]. However, the approaches do not utilize significant components of rich discriminatory information available in iris patterns.

Therefore, in order to extract rich distinctive iris features robust to contrast and brightness differences in an image or between images, the proposed method attempts to combine the two separate approaches, in which one is robust to changes in 
illumination and contrast; and the other represents rich information of iris patterns in another form. Since combing two matchers increases the complexity of the system, it is important to design an efficient way of sharing the common information from the two feature extractors as much as possible and to find a combining method that maximizes the advantage of each method.

The two methods used in this paper consider the directionality of iris patterns as a key feature and both methods decompose an iris image into 8 directional subband images using a directional filter bank (DFB) [5]. Thereafter, one of them generates a feature vector consisting of the sampled and binarized subband outputs [6], and the other takes the normalized energy values of the tessellated directional subband blocks as a feature vector [7].

Matching is performed separately between the input and template feature vectors extracted from the same feature extractor and the final decision is made by combining the two matching scores based on the accuracy of each method. Since both the two matchers extract iris features from the subband outputs decomposed by the same DFB, the complexity of the entire system does not increase so much though two matchers are combined, whereas the accuracy (or reliability) of the system increases reasonably.

\section{Iris Region Detection}

An iris is a ring shaped area surrounding the pupil of the eye as shown in Fig. 1(a). Since the pupil area has little discriminatory information, only the iris region is used for verification. Fortunately, the iris region is darker than the (white) sclera and brighter than the pupil except for eyes with cataract, thus the iris region can be easily detected by the circular edge detector [1]. Among the detected region, only the inner half regions of the left and right 90 degree cone-shaped areas are used for feature extraction in order to simply exclude the region commonly occluded by the eyelids (refer to Fig. 1(b)). The detected ROI (region of interest) is converted again into polar coordinates to facilitate the following feature extraction as illustrated in Fig. 1(c).

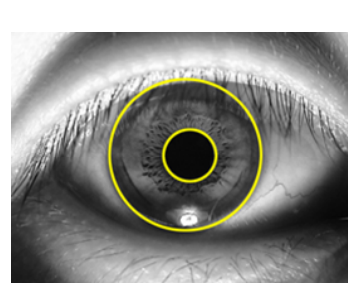

(a)

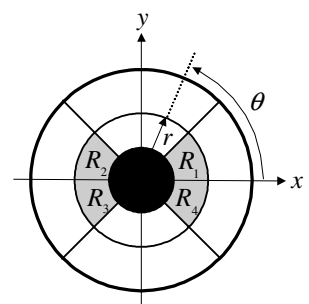

(b)

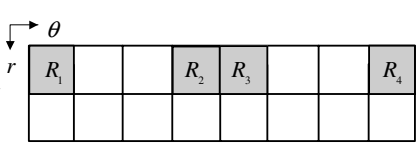

(c)

Fig. 1. Iris region detection and ROI extraction. (a) Detected inner and outer boundaries of an iris, (b) ROI in Cartesian coordinate system, and (c) ROI (R1, R2, R3, R4) in polar coordinate system. 


\section{Multimodal Directional Iris Feature Extraction}

Irises include various (directional) patterns such as arching ligament, crypts, ridges, and a zigzag collarette, thus the information on how much components of a certain direction exist according to the image location can be exploited as a good feature. For this reason, the DFB that effectively and accurately decomposes an image into several subband images is suitable for extracting directional features of iris images. The proposed method attempts to accomplish the higher accuracy by extracting and combining the two different forms of directional features (complementary features) from the directional subband outputs decomposed by the DFB.

\subsection{Directional Decomposition}

In the proposed method, the ROI images $\mathrm{R}_{1}, \mathrm{R}_{2}, \mathrm{R}_{3}$, and $\mathrm{R}_{4}$ (See Fig. 1) are decomposed into 8 directional subband outputs separately using the 8-band DFB. Since the DFB partitions the two-dimensional spectrum of an image into wedge-shaped directional passband regions accurately and efficiently as shown in Fig. 2(a), each directional component or feature can be captured effectively in its subband image. The decomposed subband images have a downscaled rectangular shape whose width and height are different and this is due to the post sampling matrices used to remove frequency scrambling [5]. Fig. 2 shows an example of the ROI images and the directional subband images decomposed by the 8-band DFB.

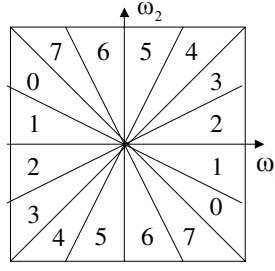

(a)

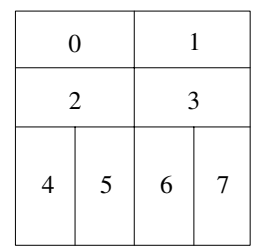

(b)

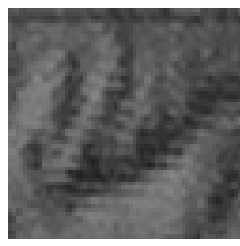

(c)

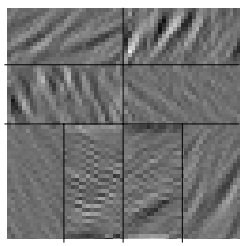

(d)

Fig. 2. Directional decomposition by the DFB. (a) Frequency partition map of the 8-band DFB, (b) positions of 8 subband outputs, (c) sample ROI image, and (d) decomposed outputs of (c).

\subsection{Binary Directional Feature Extraction}

Since the iris images are acquired by a digital camera under various internal and external illumination conditions, they have contrast and brightness differences in an image or between images. Therefore, robust features to such differences need to be extracted for reliable verification or identification. To extract the iris features that represent well the directional diversity of an iris pattern and have robustness to various brightness or contrast changes at the same time, the proposed method binarizes the directional subband outputs by making all the outputs with the positive value a binary 1 , all other outputs a binary 0 [6]. Since each decomposed subband output value has an average value of almost 0 , those values thresholded by 0 preserve the directional linear features and are robust to changes in illumination or brightness. 
The method uses an additional low-pass filter to extract the iris features on multiple scales [8]. The extracted ROI is low-pass filtered and decomposed by an 8-band DFB. The resultant subband outputs are then thresholded at either 1 or 0 according to their signs and sampled at regular intervals. For the subband outputs of an image filtered by a low-pass filter with a cut-off frequency of $\pi / n$, sampling is performed every $n$ pixels. The used method extracts the features from the two different scales, and the procedures for the feature extraction are illustrated in Fig. 3. The feature values are graphically displayed and enlarged to the original image scale to make the feature extraction procedure understandable.

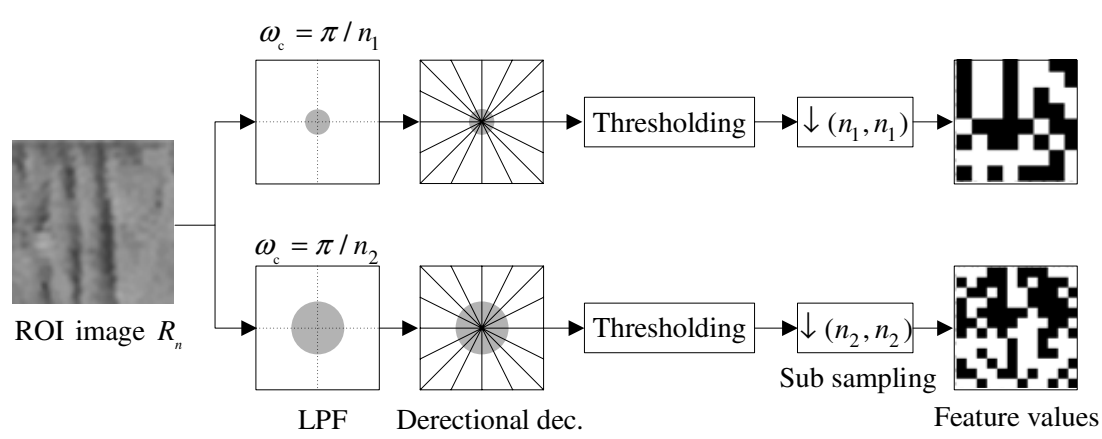

Fig. 3. Procedure for extracting the thresholded directional subband output feature

\subsection{Directional Energy Feature Extraction}

The binarized directional subband output features are robust to contrast or illumination change, however it dose not represent enough the rich information of iris patterns. Accordingly, the second method extracts another complementary feature from the directional subband outputs [7]. The intuitive feature that can be extracted from the directionally decomposed subband images is a directional energy. This directional energy can be a good feature in case that the illumination or contrast conditions are similar, but it changes severely according to illumination or contrast. Therefore, image normalization is necessary in order to use a directional energy as the iris feature, yet this is not easy in the iris image in which the brightness or contrast differences in an image or between images exist. To solve this problem, the proposed method first enhances the iris image using the method in [9] and employs the ratio of the directional energy in each block instead of the directional energy itself.

Let $e_{k \theta}{ }^{(n)}$ denote the energy value of subband $\theta$ (which we call $S_{k}{ }^{(n)}$ ). More specifically, $S_{k \theta}{ }^{(n)}$ corresponds to $k$ th block $B_{k}^{(n)}$ of the $n$th ROI image $R_{n} ; \hat{e}_{k \theta}{ }^{(n)}$ is the normalized energy value of $e_{k \theta}{ }^{(n)}$; and $c_{k \theta}{ }^{(n)}(x, y)$ is the coefficient value at pixel $(x, y)$ in subband $S_{k \theta}{ }^{(n)}$. Now, $\forall n \in\{0,1,2,3\}, k \in\{0,1,2, \ldots, 35\}$, and $\theta \in\{0,1,2, \ldots, 7\}$, the feature value, $v_{k \theta}{ }^{(n)}$, can be given as

$$
v_{k \theta}^{(n)}=\left[v_{\max } \times \hat{e}_{k \theta}^{(n)}\right]
$$


where

$$
\begin{aligned}
\hat{e}_{k \theta}^{(n)} & =\frac{e_{k \theta}^{(n)}}{\sum_{\theta=0}^{7} e_{k \theta}^{(n)}} \\
e_{k \theta}^{(n)} & =\sum_{x, y \in S_{k \theta}}\left|c_{k \theta}^{(n)}(x, y)-\bar{c}_{k \theta}^{(n)}\right|
\end{aligned}
$$

$[x]$ is the function that returns the nearest integer to $x, \bar{c}_{k \theta}{ }^{(n)}$ is the mean of pixel values of $c_{k \theta}{ }^{(n)}(x, y)$ in the subband $S_{k \theta}{ }^{(n)}$, and $v_{\max }$ is a positive integer normalization constant. In this method, the high frequency components are removed by the low pass filter to reduce the effect of noise, and then the normalized directional energy features are extracted from the low pass filtered image (see Fig. 4).

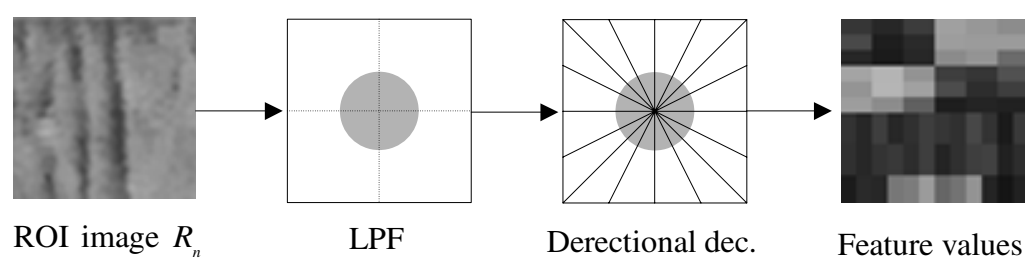

Fig. 4. Procedure for extracting the normalized directional energy feature

\section{Matching}

The two kinds of feature vectors are obtained for the single input image. One is the feature vector that consists of the binarized and sampled directional subband outputs on multiple scales, and the other is the feature vector of which elements are the blockwise normalized directional energy values. We call the former the binary feature vector, and the latter the energy feature vector for convenience' sake in this paper. In multibiometric approaches, the information presented by multiple traits can be fused at various levels such as feature extraction, matching score, and decision [10], but since the binary and energy feature vectors have different size and characteristics, combing the two feature vectors at the matching score level is one of the most effective and simplest ways. In the database, two kinds of feature vectors are also enrolled. Matching is performed between the input and template feature vectors extracted from the same feature extractor, and the final decision is made based on combining the matching scores from the two matchers.

To achieve the rotational alignment between the input and template feature vectors, the proposed method generates the additional feature vectors, in which various rotations are considered, by shifting the directional subband images and recalculating the feature values. Thereafter the method finds the minimum distances between the corresponding feature vectors for the rotational alignment $[6,7]$. 
The matching between the binary feature vectors of the input and template iris images is based on finding the Hamming distance. Let $V_{B j}{ }^{R}$ denote the $j$ th feature value of the input binary feature vector considering $R \times 45 \times(4 / N)$ degree rotation and let $T_{B j}$ denote the $j$ th feature value of the template binary feature vector, then the Hamming distance between input and template binary feature vectors, $D_{B}$, is given by

$$
D_{B}=\min _{R} \frac{1}{N_{B}} \sum_{j=1}^{N_{B}} V_{B j}^{R} \oplus T_{B j}
$$

where $R \in\{-10,-9, \ldots,-2,-1,0,1,2, \ldots, 9,10\}, N_{B}$ is the size of the binary feature vector, and $\oplus$ is an exclusive-OR operator that yields one if $V_{B j}{ }^{R}$ is not equal to $T_{B j}$, and zero otherwise.

The matching between the energy feature vectors of the input and template iris images is based on finding the Euclidean distance. Let $V_{E j}{ }^{R}$ denote the $j$ th feature value of the input energy feature vector considering $R \times 45 \times(4 / N)$ degree rotation and let $T_{E j}$ denote the $j$ th feature value of the template energy feature vector, then the Euclidean distance between input and template energy feature vectors, $D_{E}$, is given by

$$
D_{E}=\min _{R} \sqrt{\sum_{j=1}^{N_{E}}\left(V_{E j}^{R}-T_{E j}\right)^{2}}
$$

where $N_{E}$ is the size of the energy feature vector. Once the two matching distances $\left(D_{B}, D_{E}\right)$ are obtained, the final distance $D_{T}$ is calculated using the following equation:

$$
D_{T}=\alpha \cdot D_{B}+\beta \cdot D_{E}
$$

where $\alpha$ and $\beta$ are weighting factors and their sum is 1 . These weighting parameters were determined considering the EER (equal error rate), a compact measure of accuracy for biometric systems, of each method. If the final distance is below a certain threshold the input iris is accepted, otherwise it is rejected.

\section{Experimental Results}

For the experiments, we acquired a total of 434 iris images from 10 persons using a digital movie camera and 50W halogen lamp. The iris images were captured from a distance about $15-20 \mathrm{~cm}$ and the light was located below the camera so that the glint only appeared in the lower $90^{\circ}$ cone of the iris. The acquired iris images were 256 grayscale images with the size of $640 \times 480$.

In order to estimate the performance as a personal verification, an EER, which is the error rate at which a FAR (false accept rate) is equal to a FRR (false reject rate), is calculated and the result was compared with that of the Gabor filter bank-based method [1]. Table 1 shows the EER for each method.

The performance of a verification system can also be evaluated using a receiver operator characteristic (ROC) curve, which graphically demonstrates how the genuine 
acceptance rate (GAR) changes with a variation in FAR. The ROC curve for the proposed method is shown in Fig. 5. We can see that the verification performance can be effectively improved by the combining the multiple matchers.

Table 1. Decidability index and equal error rate for each method

\begin{tabular}{lcccc}
\hline Features & Gabor & Binary & Energy & Binary \& Energy \\
\hline EER & $4.25 \%$ & $5.45 \%$ & $3.80 \%$ & $2.60 \%$ \\
\hline
\end{tabular}

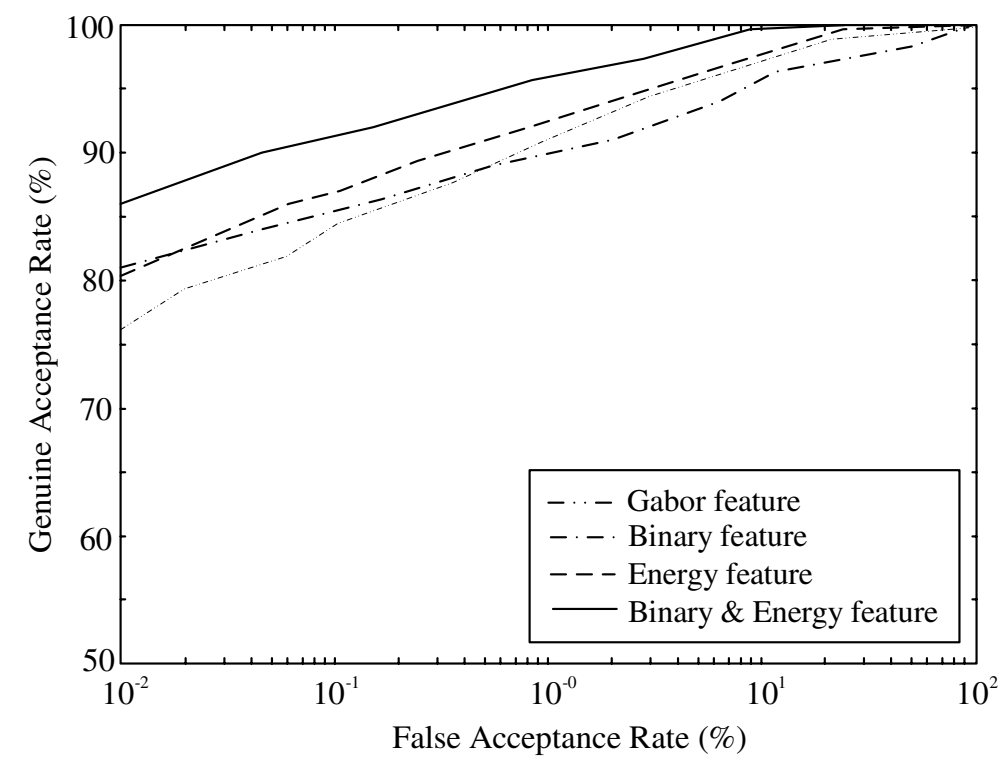

Fig. 5. ROC curve for the proposed method

\section{Conclusion}

We have presented an iris-based personal authentication method based on combining the multiple matchers. The proposed method represents the diverse directionality of the iris pattern into two forms using the same DFB: One is the binarized directional subband outputs at multiple scales, and the other is the blockwise normalized directional energy values. The former captures the multiscale and directional features that are robust to contrast or brightness differences between images, and the latter extracts another form of discriminatory iris features. Those two feature vectors are generated from the input iris image, and these feature vectors are compared with the enrolled template feature vectors, which consist of two sorts of feature vectors as in the input feature vectors. The final distance is obtained combing the matching distances from 
the two matchers. The experimental results show that the proposed multimodal approach based on combing the multiple matchers is effective in extracting robust and discriminatory iris features.

\section{Acknowledgements}

This work was supported by the IT postdoctoral fellowship program of the Ministry of Information and Communication (MIC), Republic of Korea.

\section{References}

1. Daugman, J. G.: High Confidence Visual Recognition of Persons by a Test of Statistical Independence. IEEE Trans. Pattern Anal. Machine Intell., Vol. 15, No. 11 (1993) 11481161

2. Wildes, R. P.: Iris Recognition: An Emerging Biometric Technology. Proc. IEEE, Vol. 85, No. 9 (1997) 1348-1363

3. Boles, W. W., Boashash, B.: A Human Identification Technique Using Images of the Iris and Wavelet Transform. IEEE Trans. Signal Processing, Vol. 46, No. 4 (1998) 1185-1998

4. Lim, S., Lee, K., Byeon, O., Kim, T.: Efficient Iris Recognition through Improvement of Feature Vector and Classifier. ETRI Journal, Vol. 23, No. 2 (2001) 61-70

5. Park, S., Smith, M. J. T., Mersereau, R. M.: Improved Structures of Maximally Decimated Directional Filter Banks for Spatial Image Analysis. IEEE Trans. Image Processing, Vol. 13, No. 11 (2004) 1424-1431

6. Park, C.-H., Lee, J.-J., Oh, S.-K., Song, Y.-C., Choi, D.-H., Park, K.-H.: Iris Feature Extraction and Matching Based on Multiscale and Directional Image Representation. Scale Space 2003, Lecture Notes in Computer Science, Vol. 2695 (2003) 576-583

7. Park, C.-H., Lee, J.-J., Smith, M. J. T., Park, K.-H.: Iris-Based Personal Authentication Using a Normalized Directional Energy Feature. AVBPA 2003, Lecture Notes in Computer Science, Vol. 2688 (2003) 224-232

8. Rosiles, J. G., Smith, M. J. T.: Texture Classification with a Biorthogonal Directional FilteBank. Proc. IEEE Intl. Conf. on Acoustics, Speech, and Signal Processing, Vol. 3 (2001) 1549-1552

9. Ma, L., Tan, T., Wang, Y., Zhang, D.: Personal Identification Based on Iris Texture Analysis. IEEE Trans. Pattern Anal. Machine Intell., Vol. 25, No. 12 (2003) 1519-1533

10. Jain, A. K., Ross, A.: Multibiometric Systems. Communications of the ACM, Special Issue on Multimodal Interfaces, Vol. 47, No. 1 (2004) 34-40 